\title{
Alternative Algorithm for Simultaneous Determinations of Components Poorly Resolved by Liquid Chromatography with Multiwavelength Detection
}

\author{
Hai-Long Wu, Yu-Qi Feng, Masami Shibukawa and Koichi Oguma ${ }^{\dagger}$ \\ Faculty of Engineering, Chiba University, Yayoi-cho, Inage-ku, Chiba 263, Japan
}

\begin{abstract}
This paper describes an alternative algorithm for a simultaneous multicomponent analysis with three-mode threedimensional array data generated by liquid chromatography with diode array detection (LC-DAD). The algorithm is mainly based on a combination of two-step Moore-Penrose pseudoinverse computations based on a truncated singular value decomposition (TSVD) and one-step direct bilinear decomposition. It is modeled with a series of pure or mixed reference samples and then used to analyze several unknown samples. The algorithm was evaluated by Monte-Carlo simulations of two synthetic LC-DAD data sets with different noise levels, and applied to the simultaneous determination of chlorobenzene and toluene by means of HPLC with a photodiode array detector. The obtained results show that the proposed algorithm is suitable for simultaneous determinations of several components, of which the chromatographic peaks and absorption spectra heavily overlap each other, provided that their three-dimensional data have trilinear properties.
\end{abstract}

Keywords Simultaneous multicomponent analysis, multiwavelength chromatogram, Moore-Penrose pseudoinverse, three-dimensional data analysis, Monte-Carlo simulation, truncated singular value decomposition

Along with the development of modern analytical instruments, for instance, hyphenated chromatography, that generate a two-dimensional matrix of data for each sample, it has become ever more important to explore different methods that can be applied to three-mode three-dimensional array data, such as that generated by liquid chromatography with diode array detection (LC-DAD). ${ }^{1,2}$

Several recent developments in the analysis of threedimensional data in the field of analytical chemistry have been cited in reviews by Geladi ${ }^{3}$ and Smilde. ${ }^{4}$ Several types of algorithms have already been proposed for calibrations using second-order data that can be used to estimate the concentration and spectral profile of each analyte. ${ }^{3-14}$ These algorithms include the generalized rank annihilation method (GRAM) ${ }^{5,6}$, a parallel factor analysis (PARAFAC) ${ }^{4,7}$, an unfolding principal component analysis (PCA) and unfolding partial least squares (PLS) ${ }^{8-10}$, trilinear decomposition (TLD) ${ }^{11,12}$ and a medium-rank second-order calibration using a restricted Tucker model. ${ }^{13,14}$ However, GRAM is constrained to use only one standard and one mixture samples. Also, PARAFAC does not always converge upon chemically meaningful solutions by alternating least squares (ALS) in the presence of a two-factor degeneracy problem. ${ }^{12,15}$ Unfolding PCA and unfolding PLS lack the basic property of PCA and PLS, namely maximum

\footnotetext{
$\dagger$ To whom correspondence should be addressed.
}

covariance between scores and the dependent variable in a trilinear sense. TLD probably yields imaginary eigenvalues when significant deviations from the model occur. ${ }^{16}$

In this paper, an alternative algorithm for a simultaneous multicomponent analysis with three-mode three-dimensional array data generated by LC-DAD is described. This algorithm is mainly based on a combination of two-step Moore-Penrose pseudoinverse computations based on a truncated singular value decomposition and a one-step direct bilinear decomposition. It is modeled with a series of pure or mixture reference samples, and then used to analyze several unknown samples. The algorithm has been evaluated by Monte-Carlo simulations of two synthetic LC-DAD data sets with different noise levels, and applied to simultaneous determinations of chlorobenzene and toluene by means of HPLC with a photodiode array detector.

\section{Theory}

A list of the notation used hereinafter is given in Appendix. Bold, lower case italic characters refer to vectors, bold italic capitals refer to two-dimensional matrices, and underlined bold italic capitals refer to three-dimensional matrices or three-dimensional arrays. 
The trilinear model for three-dimensional arrays, such as those generated by HPLC-DAD

Mathematical resolutions have been attempted for poorly resolved chromatographic peaks of multicomponent samples since multichannel detectors were developed. In 1984, Hoshino et al. ${ }^{17,18}$ applied a 32channel multiwavelength UV detector equipped with a data processor for a quantitative analysis of multicomponent samples, e.g., anaesthetic (ketamine) and its metabolite (nor-ketamine) in rabbit serum, naphthalene and anthracene and so on. However, much attention was not given to their earlier work. A primary reason is probably that no bilinear property present in twodimensional chromatograms was pointed out. Furthermore, their method could be modeled only with a pure component, and then applied to a quantitative multicomponent analysis of only one sample at a time; their computation method did not have sufficient numerical stability for processing the data from heavily overlapped two-dimensional chromatograms. However, the bilinear property of these two-dimensional data is very important for the development of various secondorder calibration methods. ${ }^{19}$ In 1990, Sanchez and Kowalski ${ }^{11}$ as well as Burdick and co-workers ${ }^{20}$ independently showed that three-dimensional data arrays in the trilinear form have unique factor analytical decompositions that directly yield the true chemical factor responsible for the data. Nowadays, for a threedimensional array, such as those generated by LC-DAD, their trilinear model can generally be given as follows:12,21

$$
\begin{aligned}
& r_{m h k}=\sum_{n=1}^{N} c_{m n} S_{h n} t_{k n}+e_{m h k} \\
& (m=1,2, \cdots, M ; h=1,2, \cdots, H ; k=1,2, \cdots, K)
\end{aligned}
$$

or

$$
\underline{R_{s}}=\sum_{n=1}^{N} c_{n} \otimes s_{n} \otimes t_{n}+\underline{E}=C_{s} \underline{T}^{\prime} S^{\prime}+\underline{E},
$$

where $\otimes$ denotes the outer product exemplified by $c_{n} \otimes s_{n}$ $\otimes t_{n}$, an $M$-by- $H$-by- $K$ array whose $m, h, k$ entry is $c_{n}[m] s_{n}[h] t_{n}[k]$, that is, $c_{m n} s_{h n} t_{k n}$. The three matrices, $C_{s}$, $\boldsymbol{S}$ and $\boldsymbol{T}$, are connected with an identity tensor of size $N$ by- $N$-by- $N$ or a superdiagonal three-dimensional array having ones on the superdiagonal and zeros elsewhere. These matrices obey the basic properties of linear algebra. $\underline{E}$ is a three-dimensional residual array of the same size as $\underline{\boldsymbol{R} s}$ in the stage of establishing a calibration model.

Obviously, a trilinear model for a three-dimensional array, such as that obtained by the HPLC-DAD system, is similar to the trilinear model used in the independently proposed methods by Harshman ${ }^{7,22}$ as well as by Carroll and Chang. ${ }^{23}$ The former paper considers the parallel factor models, or PARAFAC, while the latter considers canonical decompositions, or CANDECOMP. For recent discussions concerning the methods in their original contexts, see refs. 7,24 and 25 .
There exist some small distinctions between Eqs. (1) or (2) and the PARAFAC/CANDECOMP model. First, Eq. (1) or (2) seems to be considered as a trilinear regression model, while the PARAFAC model is often used for a 3-mode factor data analysis. Second, each term in Eq. (1) or (2) has a real meaning associated with the measurement system; that is, $C, S$, and $T$ denote the concentration, sensitivity coefficient and dimensionless time profile matrices corresponding to the $\mathrm{N}$ chemical components, respectively, while the solution obtained by the PARAFAC model is only partially unique, i.e., only profiles corresponding to each component are uniquely determinable. It is not difficult to understand it based on the results of Kruskal. ${ }^{26}$ Third, in Eq. (1) or (2), no side conditions, such as orthogonality, are required. However, in the PARAFAC model the condition that the vector for each dimensional be an orthogonal set is frequently imposed. Theoretically, the side condition for the PARAFAC model, such as orthogonality, is unnecessary. If this side condition is added, it will be appropriate to convert the PARAFAC trilinear model into a quadrilinear model with a core matrix, similar to the model of the 3-mode principal component analysis. ${ }^{27,28}$ For convenience, we rewrite the trilinear model in terms of matrices.

The trilinear model expressed by Eq. (1) or (2) can be written in terms of $K M$-by- $H$ matrices $\boldsymbol{R} s_{k}$ as follows:

$$
\boldsymbol{R} \boldsymbol{s}_{k}=\sum_{n=1}^{N} \boldsymbol{c}_{n} \otimes \boldsymbol{s}_{n} \boldsymbol{t}_{n}[k]+\boldsymbol{E}_{k}=\sum_{n=1}^{N} \boldsymbol{c}_{n} \boldsymbol{t}_{n}[k] \boldsymbol{s}_{n}{ }^{\prime}+\boldsymbol{E}_{k}=\boldsymbol{C} \boldsymbol{s} \boldsymbol{T}_{k} \boldsymbol{S}^{\prime}+\boldsymbol{E}_{k}
$$

Equation (3) can be further summarized as the following trilinear matrix equations (Eq. (4)):

$$
\boldsymbol{R} \boldsymbol{s}_{k}=\boldsymbol{C} \boldsymbol{s} \boldsymbol{T}_{k} \boldsymbol{S}^{\prime}+\boldsymbol{E}_{k} \quad \text { for } k=1,2, \cdots, K
$$

or the following block matrix Eq. (5):

$$
\left(\begin{array}{c}
\boldsymbol{R}_{l} \\
\boldsymbol{R}_{2} \\
\vdots \\
\boldsymbol{R}_{k}
\end{array}\right)=C \boldsymbol{s}\left(\begin{array}{c}
\boldsymbol{T}_{l} \\
\boldsymbol{T}_{2} \\
\vdots \\
\boldsymbol{T}_{k}
\end{array}\right) \boldsymbol{S}^{\prime}+\left(\begin{array}{c}
\boldsymbol{E}_{l} \\
\boldsymbol{E}_{2} \\
\vdots \\
\boldsymbol{E}_{k}
\end{array}\right)
$$

or

$$
R p=C s T p S^{\prime}+E p .
$$

Note that the subscript $(p)$ in Eq. (6) indicates a twodimensional partitioned matrix. For only one sample $(m=1)$, the following equation can be obtained:

$$
r_{l h k}=\sum_{n=1}^{N} c_{I_{n}} s_{h n} t_{k n}+e_{l h k}
$$

where $h=1,2, \cdots, H$ and $k=1,2, \cdots, K$.

Multivariate calibration model comprising two trilinear regression models similar to the PARAFAC model

Multivariate calibration generally consists of both calibration and prediction stages. ${ }^{29}$ Similarly, a second- 
order calibration may also be considered to involve two stages: an estimation of the model parameters and a prediction of the analyte concentrations in an unknown sample. For three-dimensional data, such as that generated by LC-DAD, the model in the calibration stage is given by $\mathrm{Eq}$. (1), and the model in the prediction stage is described by

$$
\begin{aligned}
& r_{l h k}=\sum_{n=1}^{N} c_{l n} s_{h n} t_{k n}+f_{l h k .} \\
& (l=1,2, \cdots, L ; h=1,2, \cdots, H ; k=1,2, \cdots, K)
\end{aligned}
$$

In matrix notation, the corresponding trilinear models can be expressed by Eqs. (4) and

$$
\boldsymbol{R} \boldsymbol{u}_{k}=\boldsymbol{C u} \boldsymbol{T}_{k} \boldsymbol{S}^{\prime}+\boldsymbol{F}_{k}, \quad \text { for } k=1,2, \cdots, K,
$$

where $\underline{F}$ denotes the three-dimensional residual array in the prediction stage and $f_{l h k}$ in (8) is its element.

In this paper, it seems to be more practical to regard the trilinear model as a trilinear regression model rather than a factor-analysis model, because (1) the concentration matrix $\boldsymbol{C s}$ in the calibration stage is practically known, (2) the time profile and sensitivity matrices, $\boldsymbol{T}$ and $\boldsymbol{S}$, in the prediction stage may also be obtained from calculations in the calibration stage by using one or more pure or mixed reference samples, and (3) theoretically the sensitivity coefficient matrix $(S)$ is not related to the elution time $(k)$.

Alternative algorithm for a second-order calibration based mainly on a combination of two-step Moore-Penrose pseudoinverse computations and one-step direct bilinear decomposition

The second-order calibration problem for several unknown samples is practically that of minimizing the value of

$$
\mathrm{f}\left(\mathrm{Cu}, \boldsymbol{T}_{1}, \boldsymbol{T}_{2}, \cdots, \boldsymbol{T}_{K}, \boldsymbol{S}\right)=\sum_{n=1}^{N}\left\|R u_{k}-C u T_{k} S^{\prime}\right\|^{2} .
$$

It may be finished sufficiently to simultaneously minimize the values of

$$
\mathbf{f}\left(\boldsymbol{C u}, \boldsymbol{T}_{k}, \boldsymbol{S}\right)=\left\|\boldsymbol{R} u_{k}-C \boldsymbol{u} \boldsymbol{T}_{k} \boldsymbol{S}^{\prime}\right\|^{2}, \quad \text { for } k=1, \cdots, K \text {. }
$$

According to this principle, an alternative algorithm for a second-order calibration based mainly on a combination of two-step Moore-Penrose pseudoinverse computations and a one-step direct bilinear decomposition is described. First, both the sensitivity coefficient matrix $(S)$ and a dimensionless time profile matrix $(T)$ are obtained by a direct bilinear decomposition of the threedimensional response array data of pure or mixed reference (standard) samples containing the chemical components of interest with the known concentration matrix $(C \mathrm{~s})$; then, both the obtained sensitivity coefficient matrix $(S)$ and the dimensionless time profile matrix $(T)$ are used to predict the corresponding com-
Table 1 Function used in the algorithm

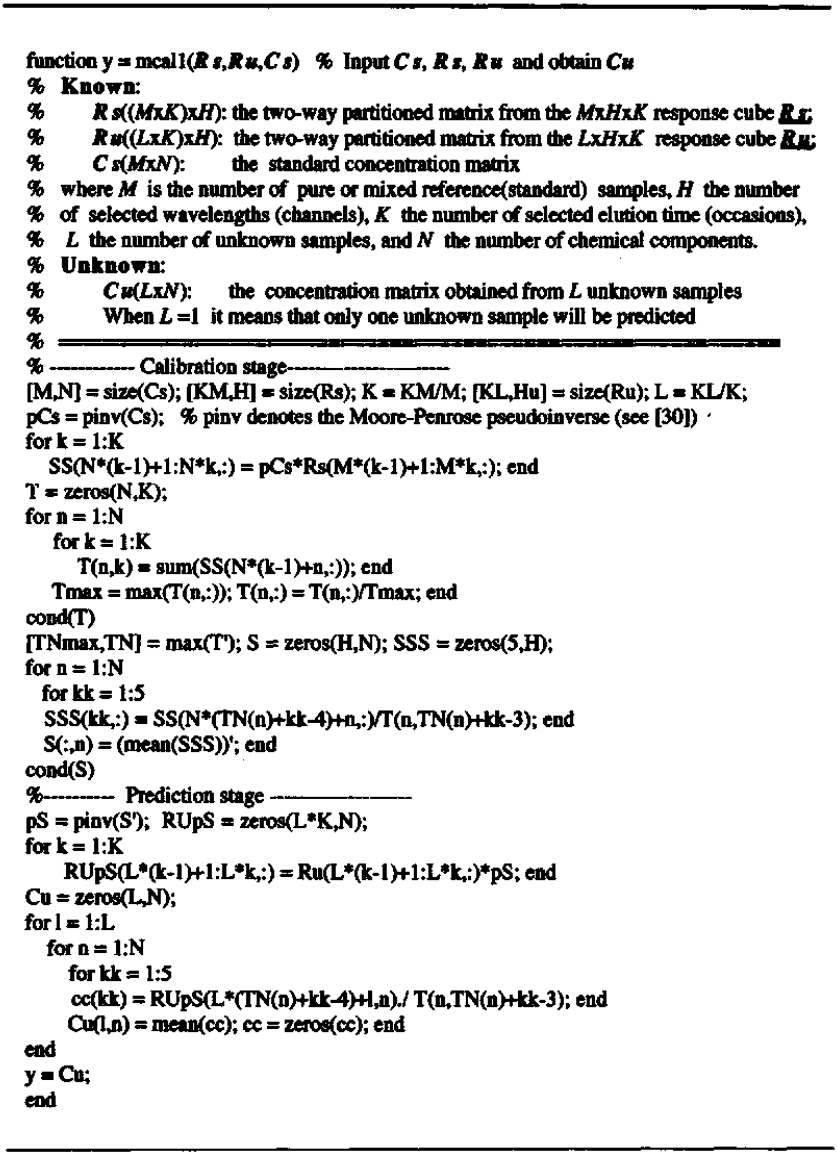

ponent concentrations of unknown samples. In the process of computing, two averaging procedures are used with a least-squares fit, averaging the estimated sensitivity matrix $(S)$ and the unknown estimated concentration matrix $\boldsymbol{C u}$ along the time axis; this increases the accuracy of the estimated results. Here, it deserves to be mentioned that in the algorithm proposed in this paper the maximum of each vector in $\boldsymbol{T}$ is kept at unity. ${ }^{26}$

The algorithm may be described as follows; also, its main part is presented in some detail in Table 1 following the style described by Golub and Vanloan. ${ }^{30}$

In the calibration stage, since $C s$ and $R s p$ are known, $T p$ and $S$ can be determined from Eq. (11). First, after calculating $M, N, H, K$, sequentially compute the $\boldsymbol{C s}^{+} \boldsymbol{R} \boldsymbol{s}_{k}$ $(k=1,2, \cdots, K)$ and obtain an $N$-by- $H$-by- $K$ threedimensional array $(\underline{D})$, that is,

$$
\boldsymbol{D}_{k}=\boldsymbol{C} \boldsymbol{s}^{+} \boldsymbol{R} \boldsymbol{s}_{k}, k=1,2, \cdots, K
$$

where the superscript $(+)$ represents the Moore-Penrose pseudoinverse. ${ }^{30,31}$ The Moore-Penrose pseudoinverse is computed by using a truncated SVD, a more robust and more accurate algorithm, with an appropriate number of principal components. The numerical stability and ruggedness of SVD have been discussed by Lawson and Hanson ${ }^{32}$ as well as by Shrager. ${ }^{33}$ It follows that $C s^{+}=\left(C s^{\prime} \boldsymbol{C s}\right)^{-1} C s^{\prime}$ and $\boldsymbol{C s}^{+} \boldsymbol{C s}$ is the $N$-dimensioned identity matrix or 
$\boldsymbol{C s}+\boldsymbol{C s}=\boldsymbol{I}_{N}$

Note that in Eq. (12) $D_{k}$ is also equivalent to $\boldsymbol{T}_{k} \boldsymbol{S}^{\prime}$. The element $d_{n h k}$ of $\underline{D}$ indicates the sensitivity coefficient value of the $n$th component at the $h$ th wavelength and the $k$ th time position.

Second, compute the dimensionless time profile matrix $(T)$, of which the element $t_{k n}$ is the $n$th diagonal element of the $k \mathrm{th}$ slice of $\underline{\boldsymbol{T}}$, as follows. For each component, calculate

$$
t_{k n}=\sum_{h=1}^{H} d_{n h k}, \quad \text { for } n=1, \cdots, N ; k=1, \cdots, K .
$$

If we let $t_{\max }(n)$ be the maximum of $\left\{t_{k n}: k=1, \cdots, K\right\}$ for the $n$th component,

$$
t_{\max }(n)=\max \left\{t_{k n}: k=1, \cdots, K\right\} \quad \text { for } n=1, \cdots, N
$$

and define the time position corresponding to $t_{\max }(n)$ as $T N(n)$, we can obtain a dimensionless time profile matrix $(T)$ by calculating

$$
\begin{gathered}
t_{k n}=t_{k n} / t_{\max }(n), \quad \text { for } k=1, \cdots, K \text { and } n=1, \cdots, N . \\
\left(t_{k n} \leq 1\right)
\end{gathered}
$$

Note that $\sum_{h=1}^{H} d_{n h k}=t_{k n} \sum_{h=1}^{H} s_{h n}(n=1, \cdots, N ; k=1, \cdots, K)$ and $\sum_{h=1}^{H} s_{h n}(n=1, \cdots, N)$, which are not related to the time $(k)$, are constant.

Since the dimensionless time profile matrix $(T)$ has been obtained, the sensitivity coefficient matrix $(S)$ can be also computed as follows:

$$
s_{h n}=d_{n h T N(n)} / t_{\max }(n), \quad \text { for } h=1, \cdots, H ; n=1, \cdots, N
$$

or

$$
\begin{aligned}
s_{h n}=[1 /(2 q+1)] \sum_{k=T N(n)-q}^{T N(n)+q} d_{n h k} / t_{k n}, \\
\\
\quad \text { for } h=1, \cdots, H ; n=1, \cdots, N
\end{aligned}
$$

where averaging of $s_{h n}$ with statistical meaning is carried out in order to improve the accuracy of the estimated results. The $q$ value is an integer that is dependent on the time interval of the three-dimensional response array data. In this paper, let $q$ be 2 , because the time interval used for an analysis of chlorobenzene and toluene is $0.02 \mathrm{~min}$.

Up to now, we have obtained the sensitivity coefficient matrix $(S)$ and the dimensionless time profile matrix $(T)$.

Similarly, in prediction stage, since $R \boldsymbol{u}_{\mathrm{p}}$ is known, we can compute $\boldsymbol{R} \boldsymbol{u}_{k}\left(\boldsymbol{S}^{\prime}\right)^{+}(k=1,2, \cdots, K)$ and obtain an $L$ by- $N$-by- $K$ three-dimensional array $\underline{\boldsymbol{A}}$ using

$$
\boldsymbol{A}_{k}=\boldsymbol{R} \boldsymbol{u}_{k}\left(\boldsymbol{S}^{\prime}\right)^{+}, k=1,2, \cdots, K,
$$

where the Moore-Penrose pseudoinverse is also com- puted by using a truncated SVD.

After finishing this, the concentration matrix $(C u)$ for $L$ unknown samples can be obtained from the following formulas:

$$
c_{l n}=a_{l n T N(n)} / t_{\max (n)}, \quad \text { for } l=1, \cdots, L ; n=1, \cdots, N
$$

or

$$
\begin{aligned}
c_{l n}=[1 /(2 q+1)] & \sum_{k=T N(n)-q}^{T N(n)+q} a_{l n k} / t_{k n}, \\
& \text { for } l=1, \cdots, L ; n=1, \cdots, N
\end{aligned}
$$

where the averaging steps in calculating the concentrations of unknown samples should be also helpful to increase the accuracy of the estimated results. Selecting of the $q$ value is done in the same way as in step 3 .

\section{Experimental}

\section{Instruments and reagents}

A high performance liquid chromatography (HPLC) system, comprising an SSC-3110T pump and an SSC3100C2 microprocessor control unit (Senshu Scientific Co., Ltd., Japan), with an L-column ODS (4.6×150 mm, Chemical Inspection and Testing Institute, Japan) and a photodiode array detector (Shimadzu SPD-M10AV) was used for simultaneous determinations of mixtures of chlorobenzene and toluene in their admixtures. The column temperature was controlled at $25.0 \pm 0.5^{\circ} \mathrm{C}$. A mixture of methanol and water $(70: 30, \mathrm{w} / \mathrm{w})$ was used as an eluent. The flow rate of the eluent was $1.0 \mathrm{ml} / \mathrm{min}$.

The response data were sampled over an elution time range of 5.50 to $6.00 \mathrm{~min}(\Delta t=0.02 \mathrm{~min})$ and wavelengths of 202.0 to $301.0 \mathrm{~nm}(\Delta \lambda=2.5 \mathrm{~nm})$. The response data matrices were collected with the help of a Compaq prolinea 4/33S personal computer with the CLASSM10A program (Shimadzu). The data taken were then transferred to a Macintosh computer (LC-III) and combined into three-dimensional response arrays, $\boldsymbol{R} \boldsymbol{s}_{\mathrm{p}}$ and $\boldsymbol{R} \boldsymbol{u}_{\mathrm{p}}$. By directly using $\boldsymbol{R} s_{\mathrm{p}}, \boldsymbol{R} \boldsymbol{u}_{\mathrm{p}}$ and $\boldsymbol{C s}$ in the algorithm presented in Table 1 , information (concentrations, relative time profiles and relative spectra) concerning unknown samples can be obtained at once.

The reagents used were of analytical grade.

\section{Algorithm programming and Monte-Carlo simulations}

The procedures described in the Theory section have been implemented in Matlab/S environment (Math Works, Inc., USA) running on a Macintosh computer. Two sets of the different multiwavelength chromatograms (LC-DAD data) with different noise levels were constructed in order to simulate a second-order calibration (see Figs. 1 and 2). The performance of the proposed algorithm was evaluated by a series of MonteCarlo simulations. ${ }^{34-36}$ Random noises with a standard deviation of $z \cdot \max \left\{r_{m h k}\right\}$ and $z \cdot \max \left\{r_{l h k}\right\}$ were added to Eqs. (5) and (8), respectively, to simulate the signal noises 

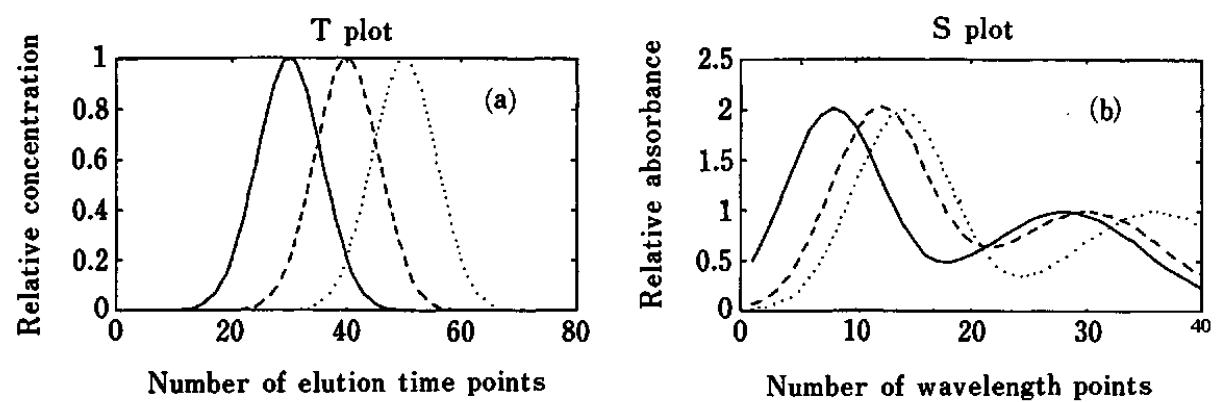

$$
\begin{array}{r}
C s=\left[\begin{array}{lll}
1.00 & 2.00 & 3.00 \\
2.00 & 1.00 & 2.00 \\
3.00 & 2.00 & 1.00 \\
2.00 & 3.00 & 1.00
\end{array}\right] \\
C u=\left[\begin{array}{lll}
1.50 & 2.50 & 2.00 \\
2.00 & 2.50 & 1.50
\end{array}\right] \\
(\text { Unit }: \mu \mathrm{g} / \mathrm{ml})
\end{array}
$$

(c)

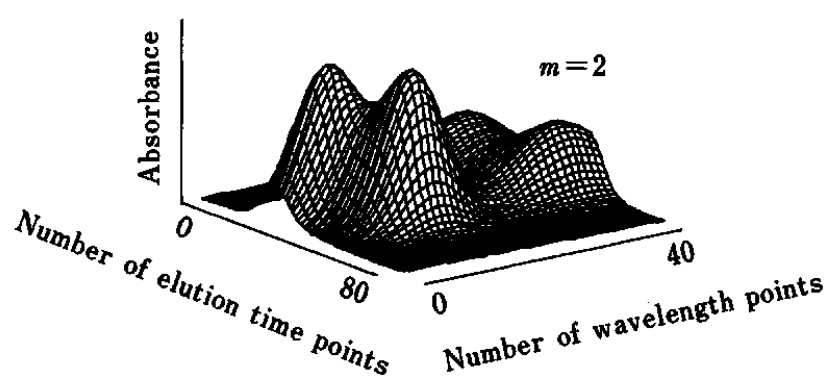

(d)

Fig. 1 Three-component simulated system (Data Set I): (a) plot of time profiles; (b) plot of sensitivity (pure spectra); (c) concentration matrices of calibration and unknown samples; (d) simulated second-order instrumental responses for a calibration sample.
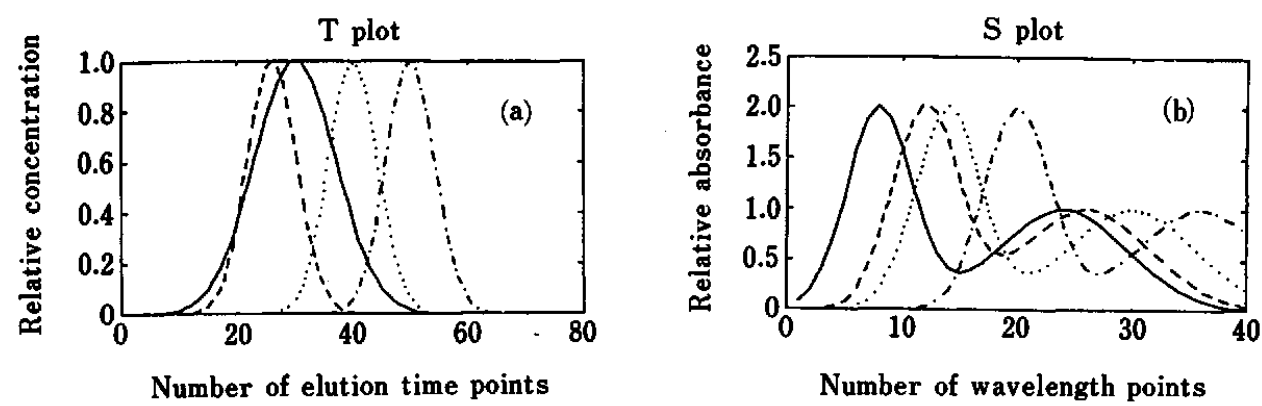

Number of elution time points

Number of wavelength points

$$
\begin{gathered}
C s=\left[\begin{array}{llll}
1.00 & 2.00 & 3.00 & 1.00 \\
2.00 & 3.00 & 1.00 & 3.00 \\
3.00 & 1.00 & 2.00 & 2.00 \\
1.00 & 3.00 & 2.00 & 3.00 \\
2.00 & 1.00 & 3.00 & 1.00 \\
3.00 & 2.00 & 1.00 & 2.00
\end{array}\right] \\
C u=\left[\begin{array}{llll}
1.50 & 2.50 & 2.00 & 1.00 \\
2.00 & 2.50 & 1.50 & 1.00
\end{array}\right]
\end{gathered}
$$

(Unit : $\mu \mathrm{g} / \mathrm{ml}$ )

(c)

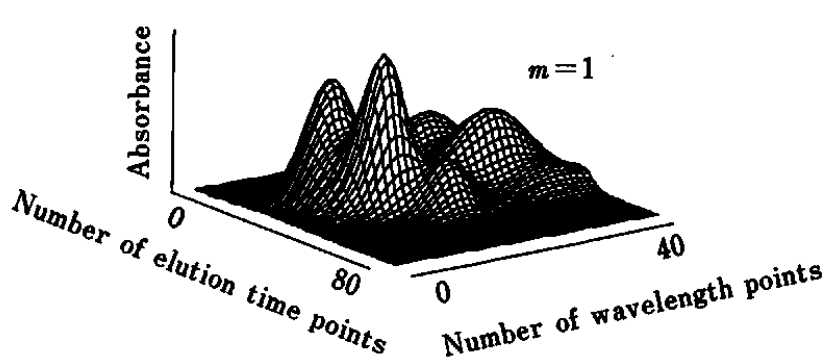

(d)

Fig. 2 Four-component simulated system (Data Set II): for (a), (b), (c) and (d) see Fig. 1.

of the analytical instrument.

Computations were iterated 50 times for each given value $z$; then, the mean and standard deviation of the root-mean-square relative predictive errors (RMSRPE) were calculated as a validatory tool for judging the predictive performance of the proposed algorithm.

$$
\operatorname{RMSRPE}=\left\{1 /(L \cdot N) \sum_{l=1}^{L} \sum_{n=1}^{N}\left[\left(c_{l n}-c_{l n}{ }^{*}\right) / c_{l n}{ }^{*}\right]^{2}\right\}^{1 / 2}
$$



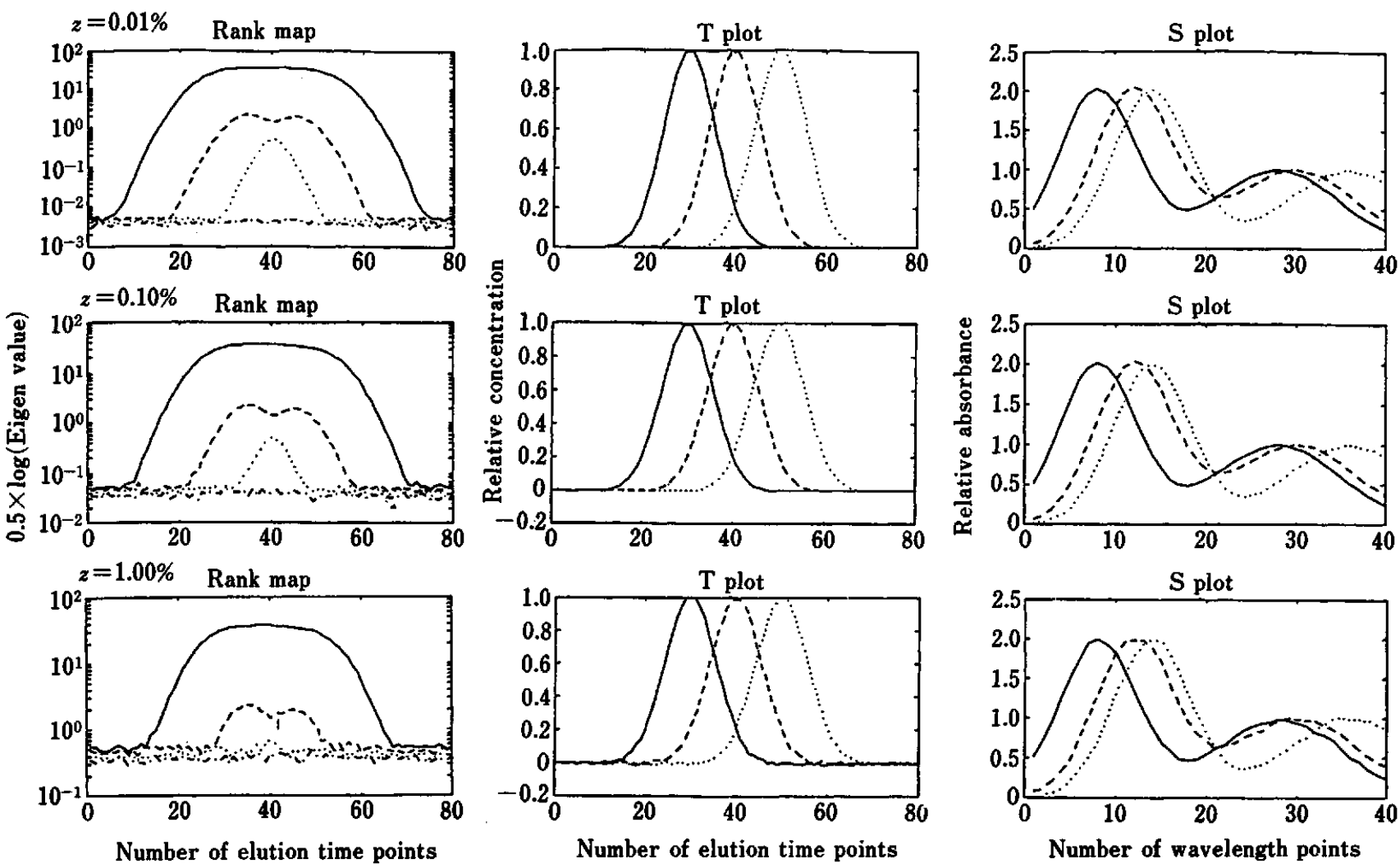

Fig. 3 Effect of the different noise levels on the results of simultaneous determinations for Data Set I.

Here, $c_{l n}$ and $c_{l n}{ }^{*}$ are the estimated and theoretical concentrations of the $n$th component in the $l$ th unknown sample, respectively.

\section{Results and Discussion}

\section{Basic performances of the algorithm}

When the given three-component and four-component simulated calibration data sets are not suffering from any noise, we can obtain not only satisfactory time profiles and spectral profiles, as shown in Figs. 1 and 2, but also their recoveries of nearly $100 \%$. In other words, when there exist no model errors and random errors, the proposed algorithm has an extremely good resolution and predictive capabilities. In order to investigate the effect of random noises on the predictive performances of the algorithm, we performed a series of Monte-Carlo simulations at different levels of random noise (see Figs. 3 and 4). The left columns in both figures are rank maps in which the eigenvalues of the $k$ th slice of $R s$ were calculated using a singular value decomposition. The middle and right columns are the relative time profiles and the relative absorption spectra obtained using the algorithms from several simulated mixed reference samples. Here, simulated mixed reference samples were adopted in order to correct the effect of interactions between the coexisting components on accuracy of the results. The means and standard deviations of the root- mean-square relative predictive errors (RMSRPE) for Data Set I and Data Set II are collected in Table 2. When $z$ is in the range from 0.01 to $1.00 \%$, the number of principal components in the rank maps are decreased from 3 to 2 for Data Set I and from 4 to 3 for Data Set II, respectively. However the resolution and predictive performances are still excellent, and the $\log$ (RMSRPE) values are nearly linear to the $\log (z)$ value. The values of $z$ reported previously are in the range from 0.01 to $0.05 \% \cdot{ }^{37}$

\section{Averaging of estimated sensitivity matrix $S$ and unknown estimated concentration matrix $\mathrm{Cu}$}

Because the three-dimensional array $\underline{D}$ in Eq. (12) and $\boldsymbol{A}$ in Eq. (19) are progressively calculated along the time axis in the proposed algorithm, respectively, it is necessary to average the estimated sensitivity matrix $(S)$ and the unknown estimated concentration matrix $(\boldsymbol{C u})$ along the time axis. The results show that the addition of both averaging steps in the algorithm is helpful to improve the performances of the algorithm.

\section{Interferences}

As reported by Booksh and Kowalski ${ }^{2}$, a second-order calibration makes it possible to detect an interferent and to perform a calibration and an analysis despite interference. In HPLC-DAD, if the interferent (of which the spectrum overlapps those of the analytes of interest) present in unknown samples can be separated 

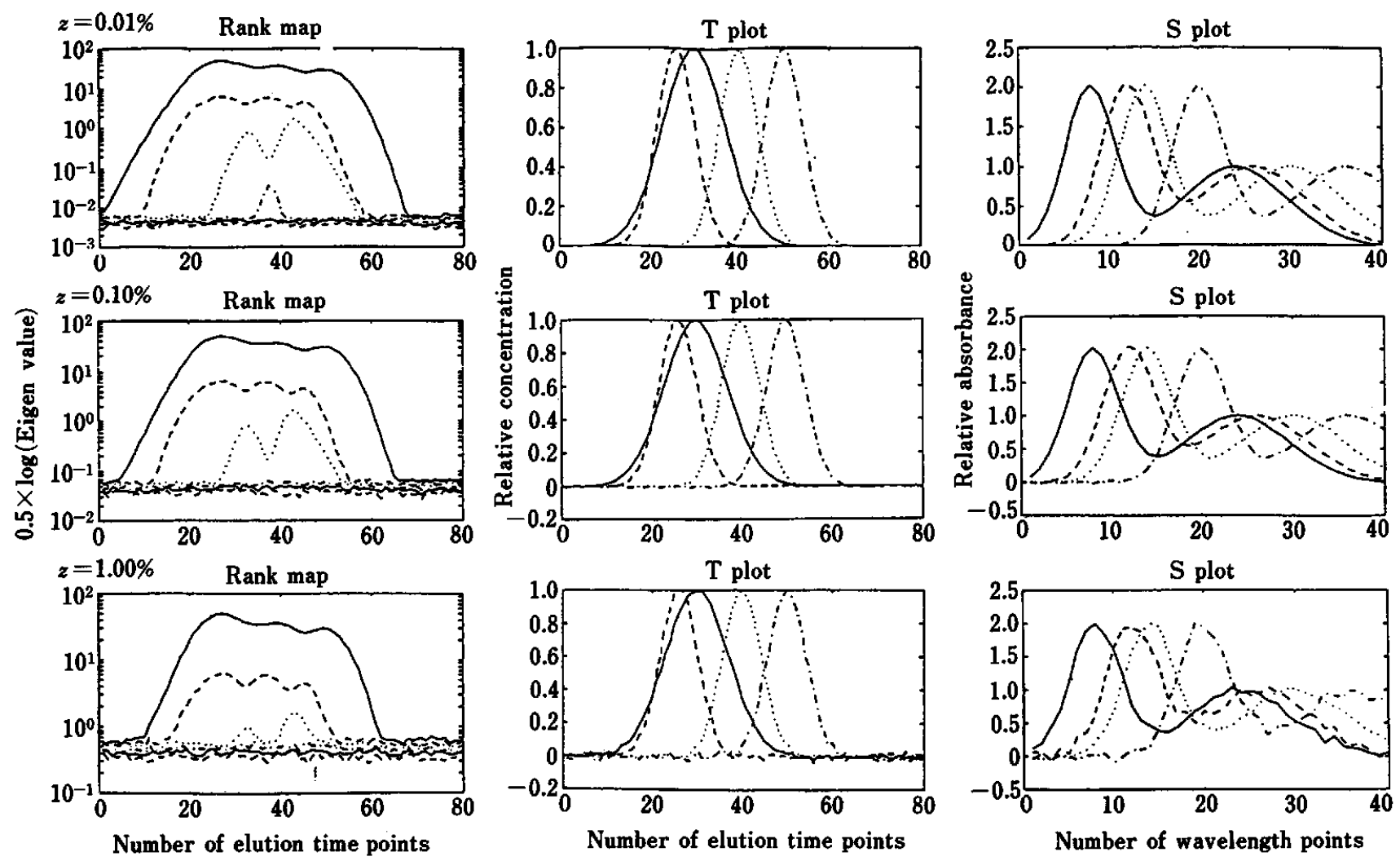

Fig. 4 Effect of the different noise levels on the results of simultaneous determinations for Data Set II.

Table 2 Effect of random noises with different levels on predictive performances of the algorithm

\begin{tabular}{|c|c|c|c|c|c|c|}
\hline \multirow{2}{*}{$\begin{array}{l}\text { Simulated three-way } \\
\text { calibration data sets }\end{array}$} & \multicolumn{6}{|c|}{$\begin{array}{l}\text { Predictive performances }\left(x_{m} \pm s\right)^{\mathbf{a}} \\
z \text { values }\end{array}$} \\
\hline & $0.01 \%$ & $0.05 \%$ & $0.10 \%$ & $0.50 \%$ & $1.00 \%$ & $5.00 \%$ \\
\hline $\begin{array}{l}\text { Three-component system } \\
\text { (Data set I) }\end{array}$ & $0.057 \pm 0.026$ & $0.360 \pm 0.164$ & $0.899 \pm 0.353$ & $3.677 \pm 1.863$ & $10.78 \pm 4.45$ & $39.16 \pm 9.53$ \\
\hline $\begin{array}{l}\text { Four-component system } \\
\text { (Data set II) }\end{array}$ & $0.068 \pm 0.038$ & $0.335 \pm 0.179$ & $0.955 \pm 0.534$ & $4.568 \pm 1.517$ & $16.88 \pm 7.58$ & $60.74 \pm 15.10$ \\
\hline
\end{tabular}

a. $x_{m}$ and $s$ are the means and the standard deviations of the root-mean-square relative predictive errors (RMSRPE). b. $z$ is a relative standard deviation relative to the maximum response value of $\underline{R} s$ or $\underline{R} \boldsymbol{u}$ as a noise level coefficient used in Monte-Carlo simulation.

from the analytes during the chromatographic process, a simultaneous multicomponent determination can be effectively carried out with the proposed algorithm, even if the chromatographic separation of the components of interest from each other is incomplete. If an unknown sample contains an interferent that cannot be separated from the analytes chromatographically, the results obtained with the algorithm will be biased. This bias depends on the correlation between the spectral profile of the interferent to each analyte and the degree of overlap in the time domain. Because the least-squares principle is adopted in this work, the solution may be considered as a unique minimal F-norm. ${ }^{30}$

The described algorithm, being used as an attempt to directly perform a multicomponent determination in the second-order sense, seems to be suitable if interferents, of which the absorption spectra are overlapped, present in the sample can be separated from the analytes during the chromatographic process or do not produce a larger bias and the three-dimensional data obtained have trilinear properties as shown below (see "simultaneous determination of chlorobenzene and toluene"). In other words, the proposed algorithm can resist a bias from the interferents to a certain degree. In cases where the interferents produce a larger bias, $T_{L D}^{11,12}$ should be considered as a good selection. However, since TLD still has the problem that it probably provides imaginary eigensolutions, it is both necessary and feasible to 
Table 3 Analytical results for a series of mixtures of chlorobenzene and toluene

\begin{tabular}{|c|c|c|c|c|c|c|}
\hline \multirow{2}{*}{ Sample } & \multicolumn{3}{|c|}{ Chlorobenzene } & \multicolumn{3}{|c|}{ Toluene } \\
\hline & Added $/ \mu \mathrm{g} \mathrm{ml}^{-1}$ & Found $/ \mu \mathrm{g} \mathrm{ml}^{-1}$ & Recovery, \% & Added $/ \mu \mathrm{g} \mathrm{ml}^{-1}$ & Found $/ \mu \mathrm{g} \mathrm{ml}^{-1}$ & Recovery, $\%$ \\
\hline$\# 1$ & 100.0 & 112.0 & 112.0 & 0.0 & -1.1 & - \\
\hline$\# 2$ & 0.0 & 0.9 & - & 100.0 & 100.8 & 100.8 \\
\hline$\# 3$ & 80.0 & 86.2 & 107.8 & 120.0 & 119.9 & 99.9 \\
\hline \#4 & 120.0 & 120.2 & 100.1 & 80.0 & 76.1 & 95.1 \\
\hline$\# 5$ & 160.0 & 151.6 & 94.8 & 40.0 & 40.3 & 100.8 \\
\hline \#6 & 80.0 & 86.7 & 108.4 & 80.0 & 80.1 & 100.1 \\
\hline
\end{tabular}
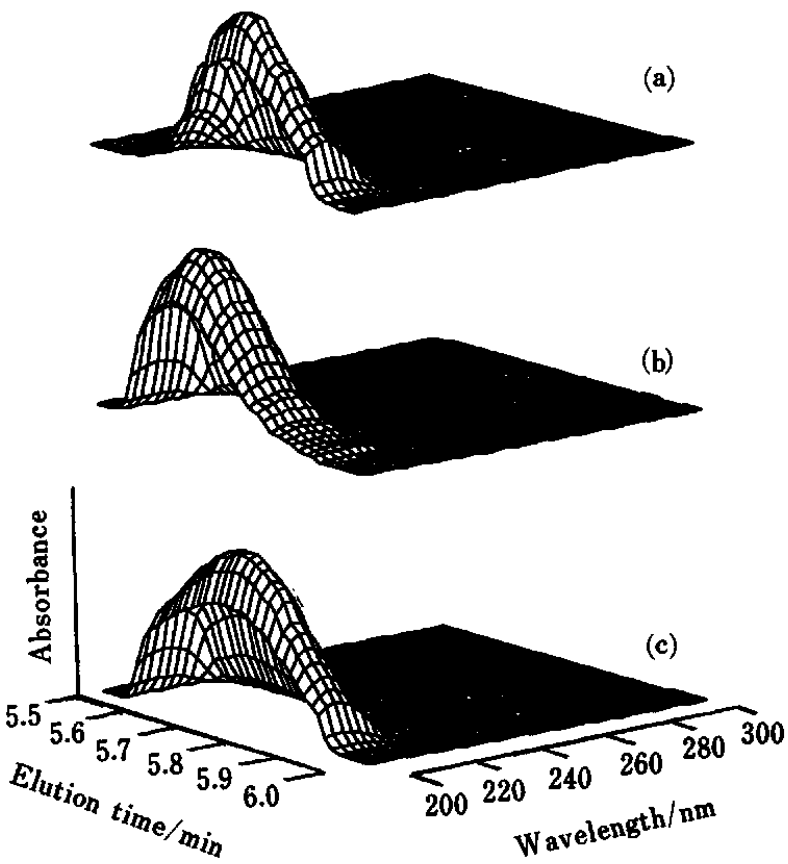

Fig. 5 Produced experimental spectrochromatograms: (a) $100.0 \mu \mathrm{g} / \mathrm{ml}$ chlorobenzene; (b) $100.0 \mu \mathrm{g} / \mathrm{ml}$ toluene; (c) a mixture solution of both $120.0 \mu \mathrm{g} / \mathrm{ml}$ chlorobenzene and $80.0 \mu \mathrm{g} / \mathrm{ml}$ toluene.

improve the TLD algorithm by combining the alternating least squares in PARAFAC with eigenanalysis-based computations in TLD according to the trilinear model.

\section{Simultaneous determination of chlorobenzene and toluene}

With the help of the HPLC system with a multichannel diode array detector, we tried to determine simultaneously chlorobenzene and toluene in their admixtures. Figure 5 reproduces the obtained instrumental responses. The three-dimensional response data were processed by the proposed algorithm; the obtained results are shown in Fig. 6 and summarized in Table 3. The retention times for chlorobenzene and toluene were $5.598 \pm 0.006 \mathrm{~min}(n=4)$ and $5.788 \pm 0.003 \mathrm{~min}(n=4)$, respectively, and the resolution of the chromatographic peaks for the two compounds was 0.33 . The condition numbers of the estimated $\mathrm{S}$ and $\mathrm{T}$ were 6.4 and 1.88 , respectively. It was shown that the spectra of chloro-
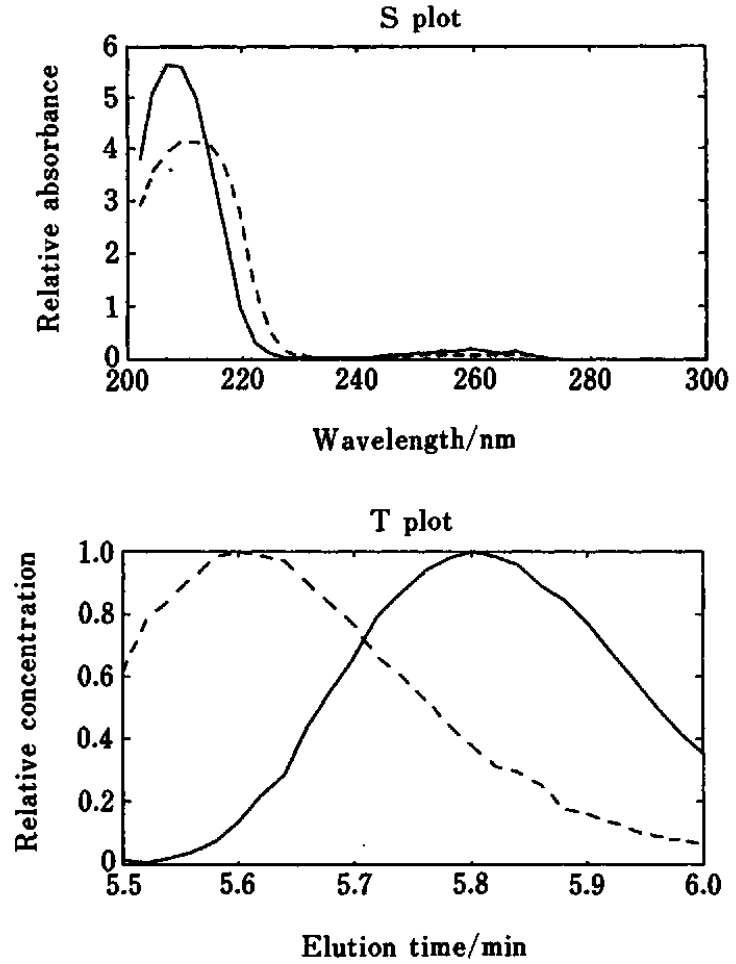

Fig. 6 Spectral profiles (sensitivity coefficient matrix $S$ ) and elution profiles (dimensionless time profile matrix $T$ ) of chlorobenzene (-) and toluene $(\cdots)$ obtained by the proposed algorithm from their mixtures.

benzene and toluene overlapped each other very heavily. The obtained recoveries were 95 to $112 \%$, and most of them are in a range of 95 to $108 \%$. The experiments also showed that the variation in the retention times of the analytes was one of the main factors that affected the accuracy of the estimated concentrations. If a suitable compound is selected and added to sample solutions as an internal standard for the retention time, the retention times observed could be effectively corrected, and more precise results might be obtained.

Some portions of this work were supported in part by the Sasakawa Scientific Research Grant from The Japan Science Society. 


\section{References}

1. T. Hirschfeld, Anal. Chem., 52, 297A (1980).

2. K. S. Booksh and B. R. Kowalski, Anal Chem., 66, 782A (1994).

3. P. Geladi, Chemom. Intell. Lab. Syst., 7, 11 (1989).

4. A. K. Smilde, Chemom. Intell. Lab. Syst., 15, 143 (1992).

5. E. Sanchez and B. R. Kowalski, Anal. Chem., 58, 496 (1986).

6. B. Wilson, E. Sanchez and B. R. Kowalski, J. Chemom., 3, 493 (1989).

7. R. A. Harshman and M. E. Lundy, Computational Statistics \& Data Analysis, 18, 15 (1994).

8. S. Wold, P. Geladi, K. Esbensen and J. Ohman, $J$. Chemom., 1, 41 (1987).

9. J. Ohman, P. Geladi and S. Wold, J. Chemom., 4, 135 (1990).

10. A. K. Smilde and D. A. Doornbos, J. Chemom., 6, 11 (1992).

11. E. Sanchez and B. R. Kowalski, J. Chemom., 4, 29 (1990).

12. K. S. Booksh, Z. Lin, Z. Wang and B. R. Kowalski, Anal. Chem., 66, 2561 (1994).

13. A. K. Smilde, Y. Wang and B. R. Kowalski, J. Chemom., 8, 21 (1994).

14. A. K. Smilde, R. Tauler, J. M. Henshaw, L. W. Burgess and B. R. Kowalski, Anal. Chem., 66, 3345 (1994).

15. E. Sanchez and B. R. Kowalski, J. Chemom., 2, 265 (1988).

16. S. Li and P. Gemperline, J. Chemom., 7, 77 (1993).

17. T. Hoshino, M. Senda, T. Hondo, M. Saito and S. Tohei, J. Chromatogr., 316, 473 (1984).

18. T. Hoshino, T. Hondo, M. Senda, M. Saito and S. Tohei, J. Chromatogr., 332, 139 (1985).

19. E. R. Malinowski, "Factor Analysis in Chemistry", 2nd ed., John Wiley \& Sons, Inc., New York, 1991.

20. D. S. Burdick, X. M. Tu, L. B. McGown and D. W. Millican, J. Chemom., 4, 15 (1990).

21. S. Leurgans and R. T. Ross, Statistical Science, 7, 289 (1992).

22. R. A. Harshman, UCLA Working Papers in Phonetics, 16, 1 (1970).

23. J. D. Carroll and J. Chang, Psychometrika, 35, 283 (1970).

24. H. Law, C. Snyder, Jr., J. Hattie and R. McDonald (ed.),"Research Methods for Multimode Data Analysis", Praeger, New York, 1984.

25. R. Coppi and S. Bolasco (ed.), "Multiway Data Analysis", Elsevier Science Publishers B.V., North-Holland, 1989.

26. J. B. Kruskal, Linear Algebra Appl., 18, 95 (1977).

27. L. R. Tucker, Psychometrika, 31, 279 (1966).

28. P. M. Kroonenberg, "Three-Mode Principal Component Analysis”, DSMO Press, Leiden, 1983.

29. H. Martens and T. Næes, "Multivariate Calibration", Wiley, New York, 1989.

30. G. Golub and C. Vanloan, "Matrix Computations", 2nd ed., The John Hopkins University Press, Baltimore, 1989.

31. H.-L. Wu, K. Oguma and R.-Q. Yu, Anal. Sci., 10, 875 (1994).

32. C. L. Lawson and R. J. Hanson, "Solving Least Squares Problems", Prentice-Hall, Englewood Cliffs, 1974.

33. R. I. Shrager, Chemom. Intell. Lab. Syst., 1, 59 (1986).

34. G. J. Hahn and S. S. Shapiro, "Statistical Models in Engineering", Section 7-3, John Wiley and Sons, New York, 1967.
35. L. M. Schwartz, Anal. Chem., 47, 963 (1975).

36. J. W. Harbaugh and G. Bonham-Carter, "Computer Simulation in Geology", R. E. Krieger Pub. Co., Malabar, 1981.

37. H. R. Keller and D. L. Massart, Anal. Chim. Acta, 246, 379 (1991).

(Received July 15, 1996)

(Accepted October 28, 1996)

\section{Appendix. Notation}

II || the Frobenius or Euclidian norm

$m$ a running index for counting reference or standard samples (objects)

$M \quad$ the number of the reference samples

$h$ a running index for counting selected wavelengths (channels)

$H$ the number of selected wavelengths (channels)

$k \quad$ a running index for counting selected elution time positions (occasions)

$K \quad$ the number of selected elution time positions (occasions)

$l$ a running index for counting unknown samples

$L \quad$ the number of unknown samples

$n$ a running index for counting chemical components of interest

$N \quad$ the number of chemical components of interest

$\underline{R}$ a three-dimensional response array from $M$ pure or mixture reference samples with a two-dimensional response matrix for each sample (size $M^{*} H^{*} K$ )

$\underline{R} \quad$ a three-dimensional response array from $L$ unknown samples (size $L^{*} H^{*} K$ )

$r_{m h k}$ the element of $\underline{\boldsymbol{R} s}$, the response of the $m$ th reference sample on the $h$ th wavelength at the $k$ th elution time position

$r_{t h k} \quad$ the element of $\underline{R} \boldsymbol{u}$, the response of the $l$ th unknown sample on the $h$ th wavelength at the $k$ th elution time position

$\boldsymbol{R} s_{k} \quad$ the $k$ th slice of $R s$ (size $\left.M^{*} H\right)$

$\boldsymbol{R} u_{k} \quad$ the $k$ th slice of $\underline{\boldsymbol{R} u}$ (size $\left.L^{*} H\right)$

Cs a concentration matrix composed of $M$ reference samples (size $M^{*} N$ )

$c_{m n} \quad$ the element of $\boldsymbol{C s}$, the concentration of the $n$th chemical component in the $m$ th reference sample

$\mathrm{Cu}$ a concentration matrix composed of $L$ unknown samples (size $L^{*} N$ )

$c_{l n}$ the element of $\mathrm{Cu}$, the estimated concentration of the $n$th chemical component in the $l$ th unknown sample

$c_{i n} * \quad$ the theorical concentration of the $n$th chemical component in the $l$ th unknown sample

$c_{n} \quad$ the $n$th column of $\boldsymbol{C s}$ or $\boldsymbol{C u}$, corresponding to the $n$th chemical component of interest (size $M^{*} l$ or $L^{*} I$ )

$S \quad$ a sensitivity coefficient matrix for $N$ chemical components of interest (size $H^{*} N$ )

$s_{n} \quad$ the $n$th column of $S$, corresponding to the $n$th chemical component of interest (size $H^{*} l$ )

$s_{h n} \quad$ the element of $S$, the sensitivity coefficient of the $n$th chemical component on the $h$ th wavelength a dimensionless time profile matrix of $N$ chemical components of interest (size $K^{*} N$ )

$\underline{T} \quad$ a three-dimensional array of size $N^{*} N^{*} K$ in which the diagonal of the $k$ th slice, $T_{k}$, is the $k$ th row of $T$

$\boldsymbol{T}_{k} \quad$ the $k$ th diagonal matrix of $\underline{T}$, whose elements are the $k$ th row of $\boldsymbol{T}$ (size $N^{*} N$ ) 
$t_{n} \quad$ the $n$th column of $T$, corresponding to the $n$th chemical component of interest (size $K^{*} I$ )

$t_{k n} \quad$ the element of $\boldsymbol{T}$

$\underline{I}$ a 3-dimensional superdiagonal array with ones on the superdiagonal and zeros elsewhere (size $N^{*} N^{*} N$ )

$\underline{E} \quad$ a three-dimensional residual array for $M$ reference samples in calibration stage (size $M^{*} H^{*} K$ )

$E_{k} \quad$ the $k$ th slice of $\underline{E}$ (size $M^{*} H$ )

$e_{m h k} \quad$ the element of $\underline{E}$

$\underline{F}$ a three-dimensional residual array for $L$ unknown samples in prediction stage (size $L^{*} H^{*} K$ )

$F_{k} \quad$ the $k$ th slice of $F$ (size $L^{*} H$ )

$f_{m h k}$ the element of $\boldsymbol{F}$

+ a notation denoting the Moore-Penrose pseudoinverse a notation denoting the transpose of a matrix

$\underline{D} \quad$ a three-dimensional array with $D_{k}=\left(C_{S}\right)^{+} R s_{k}$ for

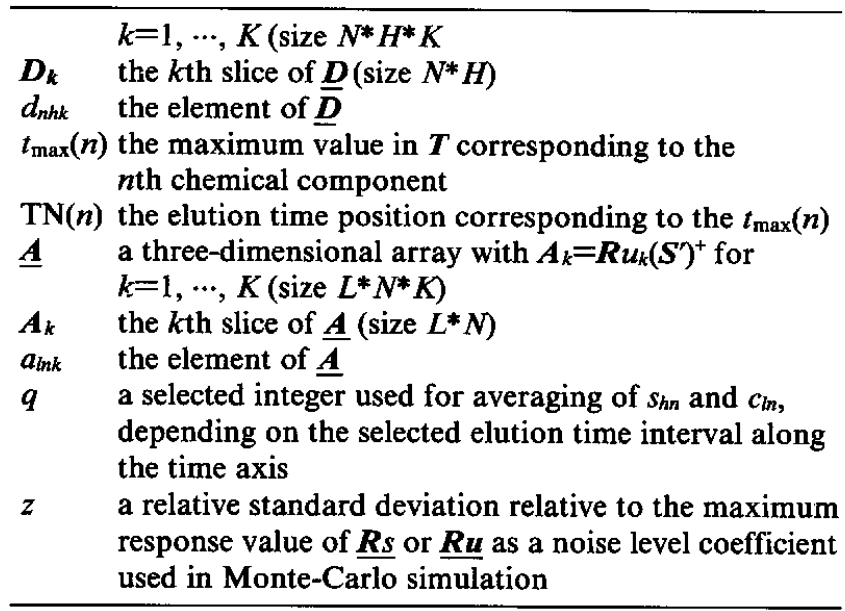

